\title{
Advice varies for suspected Lyme disease
}

A sk Laura Weeks the best way to remove a tick, and she names the options. There's the traditional straight-on tweezer pull, the counter-clockwise twist with a special tick-removal device, or popular folk remedies like rubbing the tick with olive oil or using dish soap applied on a cotton ball before covering the tick with a Band-Aid.

"I've done a lot of research," says Weeks, who is a senior research fellow with an integrative oncology clinic.

But Weeks isn't researching tick removal as part of her job. She's obsessed with ticks because she lives in Perth, Ontario, currently a hotbed of tickborne bacterial infection such as Lyme disease, caused by Borrelia burgdorferi.

"There are a few people in the community who have been affected by Lyme quite severely," says Weeks. "When you see people unable to work and there's no treatment, it's worrisome."

In fact, antibiotics are an effective treatment for Lyme disease, particularly in its early phrases. However, inadequate testing for the bacteria, confusion over what — if anything — to do in the immediate aftermath of a tick bite, and uncertainty about which ticks transmit infection are contributing to the concerns in rural areas where Lyme is increasing.

Once endemic to the United States, Europe and parts of Asia, Lyme is now well-established in British Columbia's southern mainland and Vancouver Island, and parts of Manitoba, Quebec, New Brunswick, Nova Scotia and southern and eastern Ontario. Ticks bearing the bacteria may also be in other parts of Canada, the Public Health Agency of Canada (PHAC) cautions.

The changing climate is partly responsible for the recent spread of Lyme disease, according to PHAC. In 2013, there were an estimated 500 cases of Lyme disease reported in Canada, up from 128 in 2009, when Lyme became a reportable disease. There are likely three times as many people with the underreported disease, says Steven Sternthal,

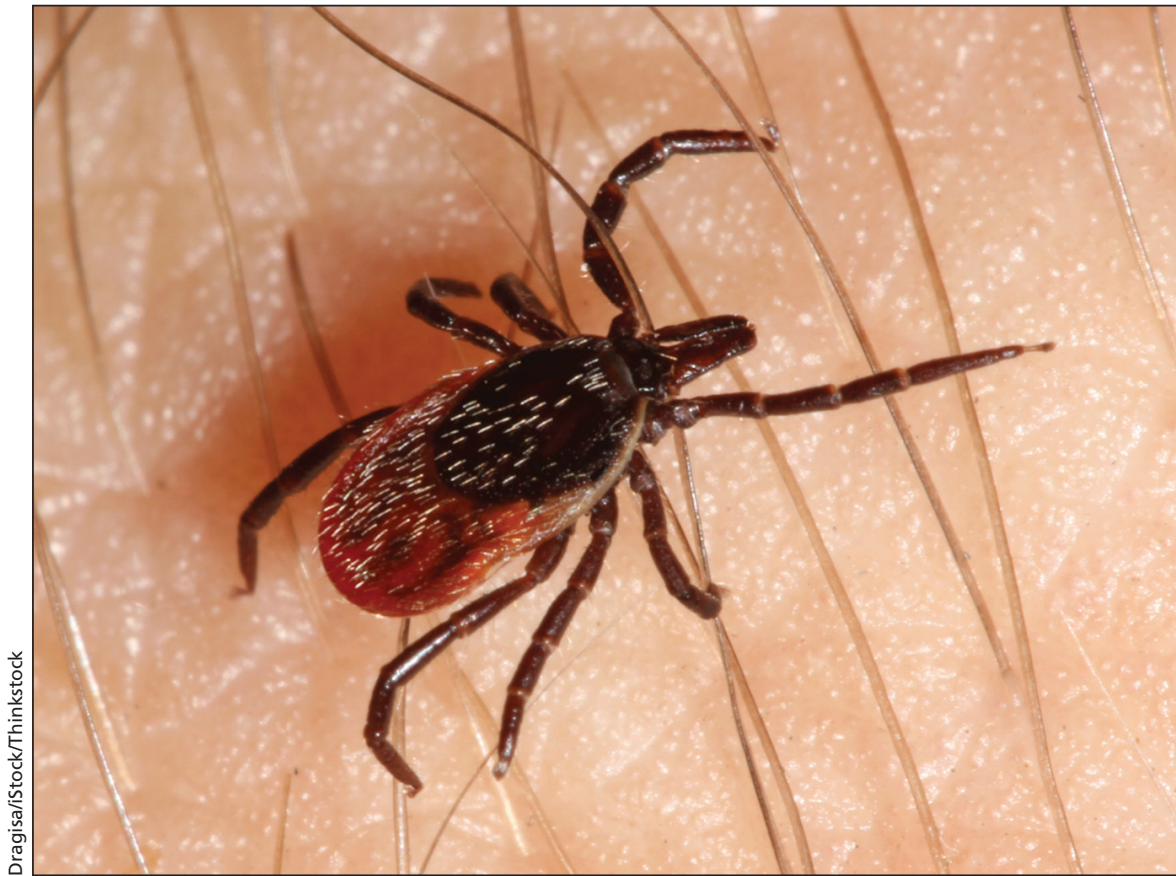

An estimated 500 cases of Lyme disease were reported in Canada in 2013, up from 128 in 2009.

acting director general of PHAC's Centre for Food-borne, Environmental and Zoonotic Infectious Diseases. The number of cases is expected to climb to more than 10000 Canadians affected annually by the 2020 s, Sternthal recently testified at the parliamentary Standing Committee on Health. There are an estimated 300000 cases per year in the US.

Not all Canadian doctors are aware that Lyme disease is even in Canada, Sternthal told the committee. The committee studied proposed legislation to create a national Lyme disease strategy, a bill currently making its way through the Senate before its expected passage later this year.

Jim Wilson, president of the Canadian Lyme Disease Foundation says medical professionals aren't getting adequate guidance about how they should treat the bite of any tick that could be carrying Borrelia. "There's an awful lot of harm being done by current [medical] practices." Medical advice varies doctor to doctor and region to region.

One Ontario patient who spoke to
CMAJ reported being told by both Telehealth and emergency department staff that he needed a tetanus shot after a tick bite. Tetanus is not an effective treatment or prevention against Lyme disease.

Other patients report being told they should have the tick tested before beginning treatment. Some patients say they were prescribed one or two doses of antibiotics immediately after a bite. Still others say doctors told them not to worry about treatment unless they exhibit symptoms.

The Canadian Lyme Disease Foundation advises that anyone bitten should remove the tick and be prescribed 21 to 28 days of a broad-spectrum antibiotic, such as doxycycline, without waiting for test results to determine whether the tick was carrying Lyme or any other infection. Wilson says the three- to four-week antibiotic regimen is standard in Europe.

Current tests may return false-negative results depending on how soon they are performed, and those available in Canada do not capture all strains of Borrelia, according to PHAC. "The 
agency is committed to improving diagnostic testing," Sternthal said.

Meanwhile, PHAC recommends that doctors diagnose on the basis of clinical assessment, rather than relying on lab tests. Once patients have early symptoms, PHAC recommends 14 to 21 days of antibiotic treatment. However, PHAC suggests that postexposure antibiotic therapy be considered in known endemic areas.
Dr. Allen Steere, the Massachusetts physician who published the first conclusive account of Lyme disease in 1977, says not all patients present with the classic bull's eye-shaped skin rash that many doctors look for to diagnose Lyme. Steere, an infectious disease specialist at the Center for Immunology and Inflammatory Diseases at Massachusetts General Hospital, believes that chronic Lyme disease exists and can result from misdiagnosis as another type of illness or lack of treatment initially.

The best advice for people is to prevent tick bites by avoiding tickinfested areas, using repellant, tucking pants into socks and wearing closed-toed shoes, and conducting daily tick checks. — Laura Eggertson, Ottawa, Ont.

CMAJ 2014. DOI:10.1503/cmaj.109-4850

\section{Portal simplifies medical registration}

$\mathrm{T}$

The Medical Council of Canada (MCC) is rolling out a national Web portal to simplify medical assessment, credentialing and registration.

Using physiciansapply.ca, medical graduates and practising physicians can register for MCC examinations, digitally share exam results and other credentials stored in the council's central repository, and request verification and translation of international credentials.

Once the system is fully deployed, users will also be able to apply for a medical licence to any of Canada's 13 provincial and territorial medical regulatory authorities through a single electronic process.

"Currently and in the past, candidates have had to apply to each province and territory individually," says MCC Executive Director Dr. Ian Bowmer. "If you wanted to apply to more than one province, you'd have to navigate the same process a number of times."

That system created unnecessary barriers for foreign doctors, "and even our own Canadian graduates, who often want to practise in more than one province early in their careers," Bowmer says.

With the new portal, "our goal was to have a one-stop application process for international medical graduates, because they needed more support than Canadian applicants," he says. "But what we've got is a system that's simpler for everyone."

In phase one of the rollout, the portal was opened to new candidates in Alberta and Nova Scotia, including Canadian medical residents, practising physicians relocating from other provinces and

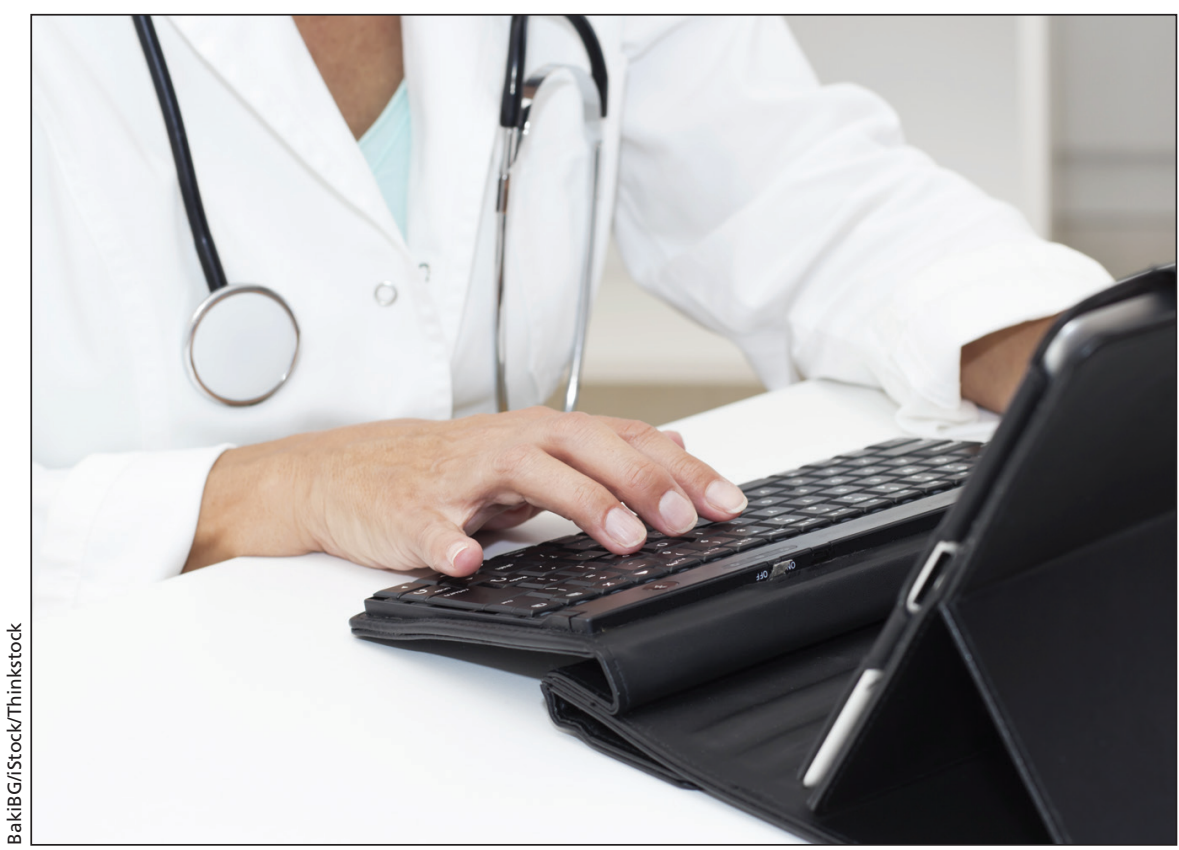

A new Web portal will let medical graduates apply for registration in multiple Canadian jurisdictions using a standardized form.

international medical graduates. Other medical regulatory authorities across Canada will adopt the new system over the next two years, says Bowmer.

Users will pay a one-time fee - $\$ 260$ in 2014 — for lifetime access to the portal. The system will also save user data to automatically populate new applications with previously entered details.

Transitioning to the new portal has been "fairly seamless," says Dr. Kate Reed, assistant registrar for the College of Physicians and Surgeons of Alberta. It helped that the province already had a fully electronic application process for medical registration, she notes.

According to Dr. William Lowe, deputy registrar for the College of Physi- cians and Surgeons of Nova Scotia, smaller jurisdictions with paper-based processes may be in for a bumpier ride. However, they may also have the most to gain from the new streamlined system.

"If it's easy to apply to five or six jurisdictions at once, you may be more likely to apply to Nova Scotia," he says. "I believe we're likely to see more applications than we would have previously.'

Although the portal will standardize the process of applying for medical registration, jurisdictions will still assess applications according to their own criteria. - Lauren Vogel, CMAJ

CMAJ 2014. DOI:10.1503/cmaj.109-4838 